\section{Histochemical detection of GM1 ganglioside using cholera toxin- B subunit. Evaluation of critical factors optimal for in situ detection with special emphasis to acetone pre-extraction}

\author{
T. Petr, ${ }^{1}$ V. Šmíd, ${ }^{1}$ J. Šmídová, ${ }^{2}$ \\ H. Hůlková, ${ }^{3}$ M. Jirkovská, ${ }^{2}$ M. Elleder, ${ }^{3}$ \\ L. Muchová, ${ }^{1}$ L. Vítek, ${ }^{1,4}$ F. Šmíd' \\ Charles University in Prague, $1^{\text {st }}$ Faculty \\ of Medicine 'Institute of Clinical \\ Biochemistry and Laboratory Diagnostics, \\ IInstitute of Histology, ${ }^{3}$ Institute of \\ Inherited Metabolic Disorders, \\ ${ }^{4} 4^{\text {th }}$ Department of Internal Medicine, \\ Prague, Czech Republic
}

\section{Abstract}

A comparison of histochemical detection of GM1 ganglioside in cryostat sections using cholera toxin B-subunit after fixation with $4 \%$ formaldehyde and dry acetone gave tissuedependent results. In the liver no pre-treatment showed detectable differences related to GM1 reaction products, while studies in the brain showed the superiority of acetone preextraction (followed by formaldehyde), which yielded sharper images compared with the diffuse, blurred staining pattern associated with formaldehyde. Therefore, the aim of our study was to define the optimal conditions for the GM1 detection using cholera toxin B-subunit.

Ganglioside extractability with acetone, the ever neglected topic, was tested comparing anhydrous acetone with acetone containing admixture of water. TLC analysis of acetone extractable GM1 ganglioside from liver sections did not exceed $2 \%$ of the total GM1 ganglioside content using anhydrous acetone at $-20^{\circ} \mathrm{C}$, and $4 \%$ at room temperature. The loss increased to $30.5 \%$ using 9:1 acetone/water. Similarly, photometric analysis of lipid sialic acid, extracted from dried liver homogenates with anhydrous acetone, showed the loss of gangliosides into acetone $3.0 \pm 0.3 \%$ only. The loss from dried brain homogenate was $9.5 \pm 1.1 \%$.

Thus, anhydrous conditions (dry tissue samples and anhydrous acetone) are crucial factors for optimal in situ ganglioside detection using acetone pre-treatment. This ensures effective physical fixation, especially in tissues rich in polar lipids (precipitation, prevention of in situ diffusion), and removal of cholesterol, which can act as a hydrophobic blocking barrier.

\section{Introduction}

Gangliosides are charged glycosphingolipids (GSLs) containing one or more sialic acid residues in their carbohydrate chains. They are highly enriched in the outer leaflet of the plasma membrane and most of their functions are associated with this location. In contrast to glycerolipids, GSLs are more rigid molecules, and therefore protect cells against harmful environmental factors by keeping the outer leaflet of the plasma membrane mechanically stable and chemically resistant. ${ }^{1-3}$ Carbohydrate moieties of gangliosides function as surface antigens, receptors for bacteria, bacterial toxins, viruses, and other bioactive molecules. Quantities and types change during development, differentiation, tumorigenesis, cell adhesion, and signal transduction. ${ }^{4}$ In situ detection of GSLs in tissue sections is important for reliable information on their subcellular distribution. GM1 ganglioside is used as the representative member of the GSL family. The detection of GM1 is based on its strong binding affinity with cholera toxin B-subunit, which is highly sensitive for GM1, thus detecting this predominantly among all other gangliosides. ${ }^{5-7}$ It can occur an additional staining due to binding of the other gangliosides, mainly GDlb, but its resolution from GM1 in histochemical detection is not possible. TLC analysis of gangliosides with resorcinol and cholera toxin staining should bring attention to unusual samples with high ratio of other gangliosides to GM1. Comparison of GM1 detection with cholera toxin and with anti-GM1 monoclonal antibody was referred by Kotani $e t$ al. ${ }^{8}$

GM1 detection is also widely used for detection of glycolipid-enriched membrane micro domains, called rafts. The fluctuations in cell surface glycolipid signaling molecules, at ultramicroscopic level, has been demonstrated in connection with fluorescence-topographic imaging at nano scale optical microscopy. ${ }^{9}$

The use of proper fixation technique is generally considered crucial for immunohistological detection of GSLs. ${ }^{10}$ Acetone fixation of cryostat sections is recommended and preferred to formaldehyde, as it provides better accessibility of antibodies or bacterial ligands to GSLs. ${ }^{10,11}$ For this reason, acetone fixation has been used in several studies. . $^{8,2,13}$

The first report on the effect of water in acetone on extraction of polar lipids was published by an author of our group..$^{14}$ In this report, it was demonstrated that the water content of acetone used during the extraction procedure, and even the water content of the treated samples, affects the degree of polar lipid extraction.

Since then, acetone fixation has been used in several studies. ${ }^{8,12,13}$ However, these studies did not mention whether water was excluded
Correspondence: František Šmíd, Associate Professor of Medical Biochemistry, Institute of Clinical Biochemistry and Laboratory Diagnostics 1st Faculty of Medicine, Charles University in Prague, Na Bojišti 312808 Prague 2, Czech Republic.

Tel. +420.224964197 - Fax: +420.224964203 .

E-mail: smid@cesnet.cz

Key words: fixation, GM1 ganglioside, cholera toxin, anhydrous acetone, $4 \%$ formaldehyde.

Acknowledgements: this research was supported by a governmental grant No. NR/9366-3 given by the Czech Ministry of Health and grant SVV-2010260512. The authors want to thank Prof. Dr. C. A. Lingwood (University of Toronto) for valuable suggestions and comments and 0 . Švejdová for technical assistance.

Received for publication: 17 November 2009. Accepted for publication: 22 March 2010.

This work is licensed under a Creative Commons Attribution 3.0 License (by-nc 3.0).

(C) Copyright T. Petr et al., 2010

Licensee PAGEPress, Italy

European Journal of Histochemistry 2010; 54:e23 doi:10.4081/ejh.2010.e23

from the acetone step, as even whether the water content of the studied sample might contribute to significant extraction of highly polar lipids. ${ }^{15}$ The aim of the present study was to define the optimal conditions for the detection of GM1 using cholera toxin B-subunit by comparing formaldehyde fixation, acetone preextraction or a combination of both (acetone + formaldehyde sequence) in tissues with different polar lipid content; in this study liver and brain were used as representative tissues with significantly different polar lipid content.

\section{Materials and Methods}

\section{Chemicals}

Paraformaldehyde, cholera toxin B-subunit biotin-labeled (ChT-B-biotin), streptavidinperoxidase-polymer, albumin, biotin, and diaminobenzidine (DAB)-tetrahydrochloride tablets were supplied by Sigma (St Louis, MO, USA); avidin was obtained from Fluka (Buchs, Switzerland); cholera toxin B-subunit conjugated with peroxidase (ChT-B-Px) came from List laboratories (USA) and DEAE Sephadex was supplied by GE (Healthcare, UK). All other chemicals were purchased locally from Penta (Czech Republic).

\section{Experimental animals}

Female Wistar rats (Anlab, Prague, Czech 
Republic) were housed under controlled temperature and a natural light-dark cycle. Liver and brain samples were taken from the Wistar rats and snap frozen.

All aspects of the study met the accepted criteria for the experimental use of laboratory animals and all protocols were approved by the Animal Research Committee of the $1^{\text {st }}$ Faculty of Medicine, Charles University, Prague, Czech Republic.

\section{Preparation of fixatives}

Formaldehyde: $4 \%$ formaldehyde was freshly prepared by depolymerization of paraformaldehyde.

Dry acetone: $1000 \mathrm{~mL}$ of acetone was dried over 100-150 g of anhydrous calcium chloride in a closed glass bottle (with occasional mixing) for several days, then decanted and distilled under elimination of air moisture. The obtained dry acetone was stored in tightly closed bottles.

\section{Quantification of GM1 ganglioside extraction with acetone from liver sections}

Twelve $6 \mu \mathrm{m}$ cryostat sections from normal rat liver were cut and placed on the same microscopic slide. One slide, containing 12 sections, represented one sample for TLC densitometry. The samples (slides) used in each extraction were in triplicates.

The loss of GM1 ganglioside during acetone fixation was studied by comparison of the following extractions:

1) The samples (slides) were extracted directly with chloroform-methanol-water (C-MW) $10: 10: 1 \mathrm{v} / \mathrm{v} / \mathrm{v}$ with shaking on an orbital shaker at room temperature $\left(25^{\circ} \mathrm{C}\right)$ for $10 \mathrm{~min}$. These samples were used to measure the total quantity of GM1 in the tissue sections.

2) Other samples were extracted with i) dry acetone at room temperature for $15 \mathrm{~min}$, ii) dry acetone at $-20^{\circ} \mathrm{C}$ for $15 \mathrm{~min}$, and iii) a cold acetone-water $9: 1 \mathrm{v} / \mathrm{v}$ mixture, at $-20^{\circ} \mathrm{C}$ for 15 min.

The samples from the acetone extraction were re-extracted with C-M-W 10:10:1 v/v/v by shaking at room temperature for $10 \mathrm{~min}$.

All extracts were evaporated under a stream of nitrogen, dissolved in chloroform-methanol $1: 1 \mathrm{v} / \mathrm{v}$, and then applied onto HPTLC aluminium sheets of silica gel (Merck; Darmstadt, Germany). Chromatograms from the experiments were developed in a solvent mixture C:M:0.2\% aqueous $\mathrm{CaCl}_{2}$ (50:45:11). After drying, the silica gel layer was impregnated with $0.1 \%$ polyisobutylmethacrylate in cyclohexane. Non-specific binding was blocked using 1\% BSA in PBS. Thereafter, the biotin-labeled cholera toxin B-subunit (diluted 1:300) was bound to the GM1 ganglioside at room temper- ature for $30 \mathrm{~min}$. After extensive washing, streptavidin-peroxidase-polymer (diluted 1:400) was used for ultra-sensitive binding to biotin and amplification of the peroxidase enzyme signal. After washing, a blue reaction product was formed by reaction of the peroxidase with a solution of 1-chloronaphtol and $\mathrm{H}_{2} \mathrm{O}_{2}$ in a citrate phosphate buffer $(\mathrm{pH}=7.2)$.

Densitometry of the chromatogram in reflectance mode at $580 \mathrm{~nm}$ (CAMAG TLC Scanner II, Switzerland) was used for evaluation of the percentage distribution of GM1, in both the acetone and C-M-W extracts.

\section{Quantification of acetone extraction of gangliosides from dried liver and brain homogenates}

A very fine-grained homogenates were prepared from $1 \mathrm{~g}$ of liver and $0.5 \mathrm{~g}$ of brain, dried using a two-day lyophilization and then rapidly transferred into desiccator (to eliminate condensation of air moisture on the cold samples). One group of samples was extracted with C-M$\mathrm{W}(4: 8: 3)$ repeated extractions $(3 \mathrm{x})$ with shaking (extracts $A, n=6$ ). The collected extracts represented the total gangliosides in the sample. The second group was extracted with dry acetone at room temperature for 15 min with shaking (extracts $B, n=6$ ) and afterwards reextracted (3x) with C-M-W (4:8:3) using the same extraction technique described for extract A (extracts $C, n=6$ ).

Gangliosides from the extracts were isolated with ion exchange chromatography (DEAE Sephadex), base treatment, dialysis and silica gel column chromatography according to the procedure described by Ledeen et al. ${ }^{16}$ Total gangliosides (total lipid sialic acid) in the extracts were determined using the resorcinolHCl method. ${ }^{17}$

\section{Histochemical detection of GM1 ganglioside in rat liver sections}

Cryostat liver sections $6 \mu \mathrm{m}$ thick were dried overnight at room temperature, and fixed with freshly prepared $4 \%$ formaldehyde at room temperature for $5 \mathrm{~min}$. The parallel sections were fixed in dry acetone at $-20^{\circ} \mathrm{C}$ for 2 or 15 min, then briefly transferred into a desiccator in order to eliminate air moisture condensation on the cold microscopic slides and thereby dilution of the acetone with condensed water. The influence of temperature on GM1 extraction from liver sections was studied by comparing fixation with dry acetone at $-20^{\circ} \mathrm{C}$ for 2 or $15 \mathrm{~min}$ and fixation at room temperature for 2 or $15 \mathrm{~min}$. The effect of the water content in the acetone fixative was studied in a further experiment comparing fixation using dry acetone with an acetone-water mixture $(9: 1 \mathrm{v} / \mathrm{v})$, both at $-20^{\circ} \mathrm{C}$ for $15 \mathrm{~min}$. After acetone fixation, liver sections were dried in desiccator at room temperature and then put into PBS.

Histochemical detection of GM1 ganglioside was performed with ChT-B-biotin, according to the procedure described by Jirkovská et al. ${ }^{18}$ In brief, the endogenous peroxidase activity was blocked by incubation in PBS, supplemented by $1 \% \mathrm{H}_{2} \mathrm{O}_{2}$ and $0.1 \%$ sodium azide. Endogenous biotin was blocked using a blocking kit (DakoCytomation, Denmark). In order to block non-specific binding, sections were treated with 3\% BSA in PBS for 15 min. Sections were then incubated with ChT-B-biotin, diluted 1:300 with PBS plus $3 \% \mathrm{BSA}$, for $16.5 \mathrm{~h}$ at $8^{\circ} \mathrm{C}$. After washing with PBS, the sections were incubated with streptavidin-peroxidase-polymer diluted 1:400 with PBS at room temperature for $60 \mathrm{~min}$. Peroxidase activity was visualized using DAB tetrahydrochloride in darkness for 20 min.

In each series two control tests were included. First, ChT-B-biotin was omitted in a negative test; second, fixed sections were extracted with a C:M (1:1 v/v) and C-M-W 1:1:0.3 v/v/v mixtures at room temperature, both for $15 \mathrm{~min}$ with shaking before detection of GM1. In order to obtain comparable results, corresponding areas were selected on parallel liver sections and photographed under constant exposure conditions.

\section{Densitometric analysis of GM1 ganglioside in tissue sections}

Liver sections obtained from 23 Wistar rats were analyzed. In each animal, the optical density of GM1 staining in formaldehyde-fixed samples represented $100 \%$ and the density of GM1 staining after acetone fixation was expressed as a proportion.

The mean optical density of the GM1 ganglioside reaction product in liver parenchyma was measured using an Olympus Cue 2 densitometric program at 70 areas in each section, at a magnification of $500 x$. Areas of sections containing either large vessels or technical artifacts (disruptions, etc.) were excluded from measurements.

\section{Comparison of histochemical detection of GM1 ganglioside in rat liver and brain sections}

Parallel cryostat sections from liver tissues were treated with the following fixatives: 1) $4 \%$ formaldehyde; 2) anhydrous acetone. Sections from brain tissue were fixed with: 1) formaldehyde; 2) Baker's solution (formaldehyde containing calcium chloride); 3) acetone followed by formaldehyde $(\mathrm{A}+\mathrm{F})$; and 4$)$ acetone followed by Baker's solution (A+B). Acetone followed by formaldehyde gave better-preserved morphology than acetone alone. After acetone fixation, liver and brain sections were dried in a desiccator, put into formaldehyde or Baker's 
A

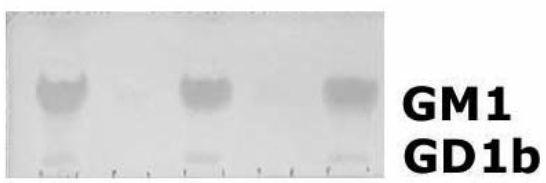

$\begin{array}{lllll}1 & 2 & 3 & 4 & 5\end{array}$
B

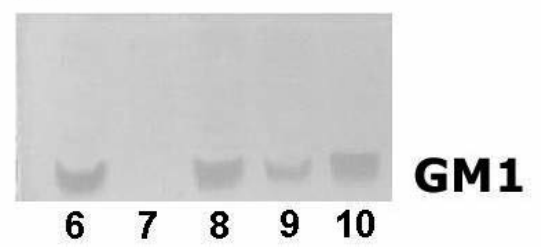

Figure 1. TLC of gangliosides extracted under various conditions. (A) Effect of temperature on dry acetone extractability of GM1 ganglioside. (1) direct extraction with C-M-W; (2) extract with dry acetone at $-20^{\circ} \mathrm{C}$; (3): C-M-W extract after previous extraction with dry acetone at $-20^{\circ} \mathrm{C}$; (4): acetone extract at room temperature; (5): C-M-W extract after previous extraction with dry acetone at room temperature. Note: GM1 ganglioside detected with ChTB (GD1b ganglioside band was also detected with much lower sensitivity). GM1 content in all acetone extracts was below the detection level of very sensitive cholera toxin staining. (B) Comparison of GM1 extraction with dry acetone and with aqueous acetone. (6) Direct extraction with C-M-W; (7) extract with dry cold acetone at $-20^{\circ} \mathrm{C}$. (8): C-M-W extract after previous extraction with dry acetone at $-20^{\circ} \mathrm{C}$; (9) extracts with mixture acetone-water 9:1 shows significant loss of GM1; (10) C-M-W extracts after previous extraction with acetone-water mixture.
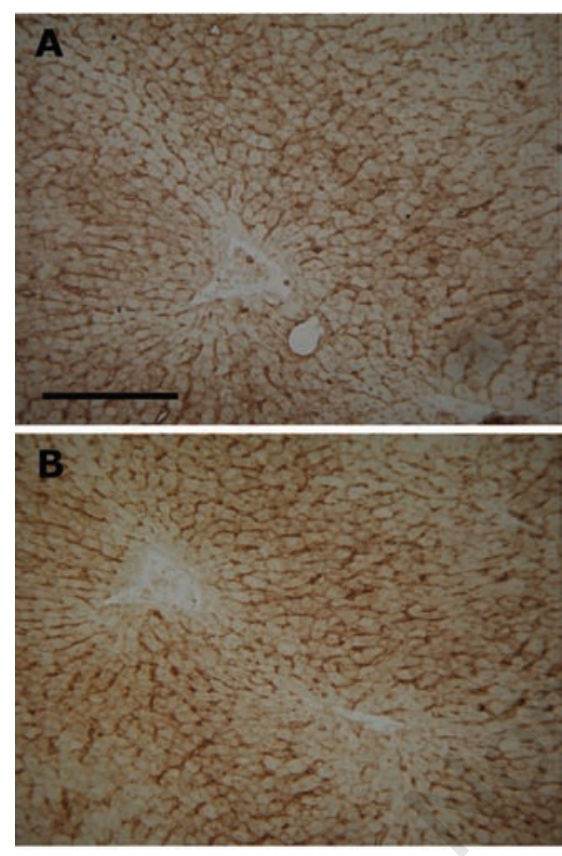

Figure 2. Histochemical detection of GM1 ganglioside in liver cryostat sections after various fixation conditions. Fixation with (A) dry cold acetone for $15 \mathrm{~min}$ at $-20^{\circ} \mathrm{C}$ and with (B) $4 \%$ formaldehyde for 5 minutes at room temperature gave comparable results. Rat liver cryostat sections stained with cholera toxin B-subunit. Bar $200 \mu \mathrm{m}$.

solution and then into PBS. Histochemical detection of GM1 ganglioside was performed using cholera toxin B-subunit labeled with peroxidase (ChT-B-Px) diluted 1:500 with PBS plus $3 \% \mathrm{BSA}$ and incubated for $16.5 \mathrm{~h}$ at $8^{\circ} \mathrm{C}$. Cell nuclei were counterstained with hematoxylin. Other steps were applied according to the previously mentioned staining protocol (see above), except for blocking of biotin.

Unmasking treatment (cholesterol removal) with methyl- $\beta$-cyclodextrin (MCD) was performed on brain sections only. $12 \mathrm{mM}$ MCD in PBS was used for $30 \mathrm{~min}$ at room temperature and for $45 \mathrm{~min}$ at $37^{\circ} \mathrm{C}$. A $20 \mathrm{mM}$ MCD concentration was used for $30 \mathrm{~min}$ at room temperature. After MCD treatment, sections were briefly washed in PBS and then fixed with formaldehyde.

\section{Determination of cholesterol}

Cholesterol was determined in the lipid extracts from both rat liver and brain samples using gas chromatography, with isotope dilution mass spectrometry, ${ }^{19}$ using D7-cholesterol as the internal standard.

\section{Statistical analysis}

SigmaStat software (Jandel Scientific) was used for statistical analyses. Data are presented as the median and IQ range, and assessed using the Mann Whitney rank sum test. A $\mathrm{P}<0.05$ was considered as statistically significant.

\section{Results}

\section{Effect of different fixation procedures on GM1 ganglioside \\ extraction from the liver sections}

The loss of GM1 ganglioside due to the fixation of dry cryostat liver sections with dry acetone was studied and compared with those samples extracted with chloroform-methanolwater 10:10:1 v/v/v (taken as 100\% of GM1). First, the effect of the temperature of dry acetone was studied at $-20^{\circ} \mathrm{C}$ and $25^{\circ} \mathrm{C}$; the sec- tions extracted with acetone were re-extracted with C-M-W 10:10:1. The results of the TLC of gangliosides in extracts stained with the cholera toxin B-subunit are shown in Figure 1A. A similar procedure was used to demonstrate the effect of water content in acetone on the extraction of gangliosides (Figure 1B).

Both chromatograms were evaluated using densitometry. Figures $1 \mathrm{~A}$ and $1 \mathrm{~B}$ illustrate that the GM1 content from dry acetone extracts was not detectable, even when the highly sensitive cholera toxin method was used (Figure 1). The greatest part of GM1 persisted after acetone fixation and extraction; and it was extracted by the $\mathrm{C}-\mathrm{M}-\mathrm{W}$ re-extraction. Therefore, the percentage of GM1 in the acetone extracts was counted as the difference between the mean density of GM1 from samples extracted directly with C-M-W 10:10:1 v/v/v (taken as 100\% of GM1) minus the mean density detected in C$\mathrm{M}-\mathrm{W}$ re-extracts. GM1 content in C-M-W reextracts was $98.1 \%$ after cold acetone, and $96.2 \%$ after acetone at $25^{\circ} \mathrm{C}$ (i.e., the loss of GM1 was $1.9 \%$ with dry acetone at $-20^{\circ} \mathrm{C}$ and $3.8 \%$ at $25^{\circ} \mathrm{C}$, respectively). On the other hand, the acetone:water mixture $(9: 1)$ yielded a remarkable $30.5 \%$ loss of GM1 (Figure 1B, line 9). The almost ten times higher extraction of GM1 in acetone:water (9:1) extract demonstrates that the water content in acetone has a significant effect on GM1 extraction.

\section{Effect of fixation procedures studied on liver and brain dried homogenates}

The semi-quantitative results obtained from the densitometry of chromatograms were supported by a quantitative determination (photometric resorcinol-HCl method) of the loss of total gangliosides from dried liver and brain homogenate into acetone.

The mean value of the amount of total gangliosides extracted with C-M-W (4:8:3) from dried liver and brain homogenate (extract A) was taken as $100 \%$ of total gangliosides. The loss of gangliosides from liver into acetone at room temperature (extract B) was $3.0 \pm 0.3 \%$ ) and from brain $9.5 \pm 1.1 \%$ and in the C-M-W reextract of liver (extract $\mathrm{C}$ ) remained $97.0 \pm 0.3 \%$ ), and in re-extracts from brain about $90.5 \%$.

\section{Histochemical detection of GM1 ganglioside in liver cryostat sections under various fixation conditions}

Comparable GM1 staining intensity and pattern were observed in liver cryostat sections after both formaldehyde and anhydrous acetone fixations (Figure 2). For dry acetone, the effects of fixation temperature and time on 


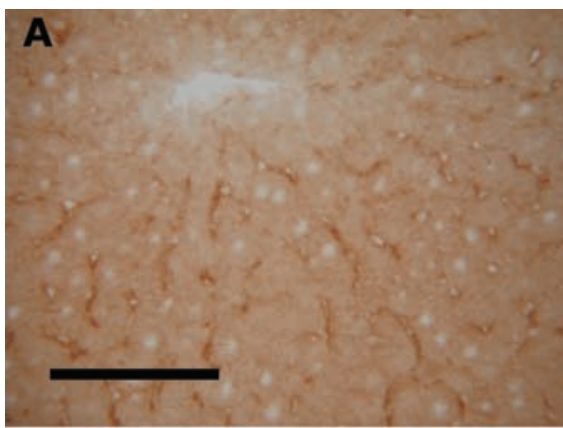

Figure 3. Effect of acetone temperature on histochemical detection of GM1 ganglioside in liver cryostat sections. Fixation with (A) $4 \%$ formaldehyde for 5 minutes at room temperature $(\mathrm{F})$; $(\mathrm{B})$ cold dry acetone for 2 minutes and $(C) 15 \mathrm{~min}$, respectively; (D) dry acetone at room temperature for $2 \mathrm{~min}$ and (E) $15 \mathrm{~min}$, respectively. No apparent differences between GM1 staining intensity or in pattern. Rat liver cryostat sections stained with cholera toxin B-subunit. Bar $100 \mu \mathrm{m}$.
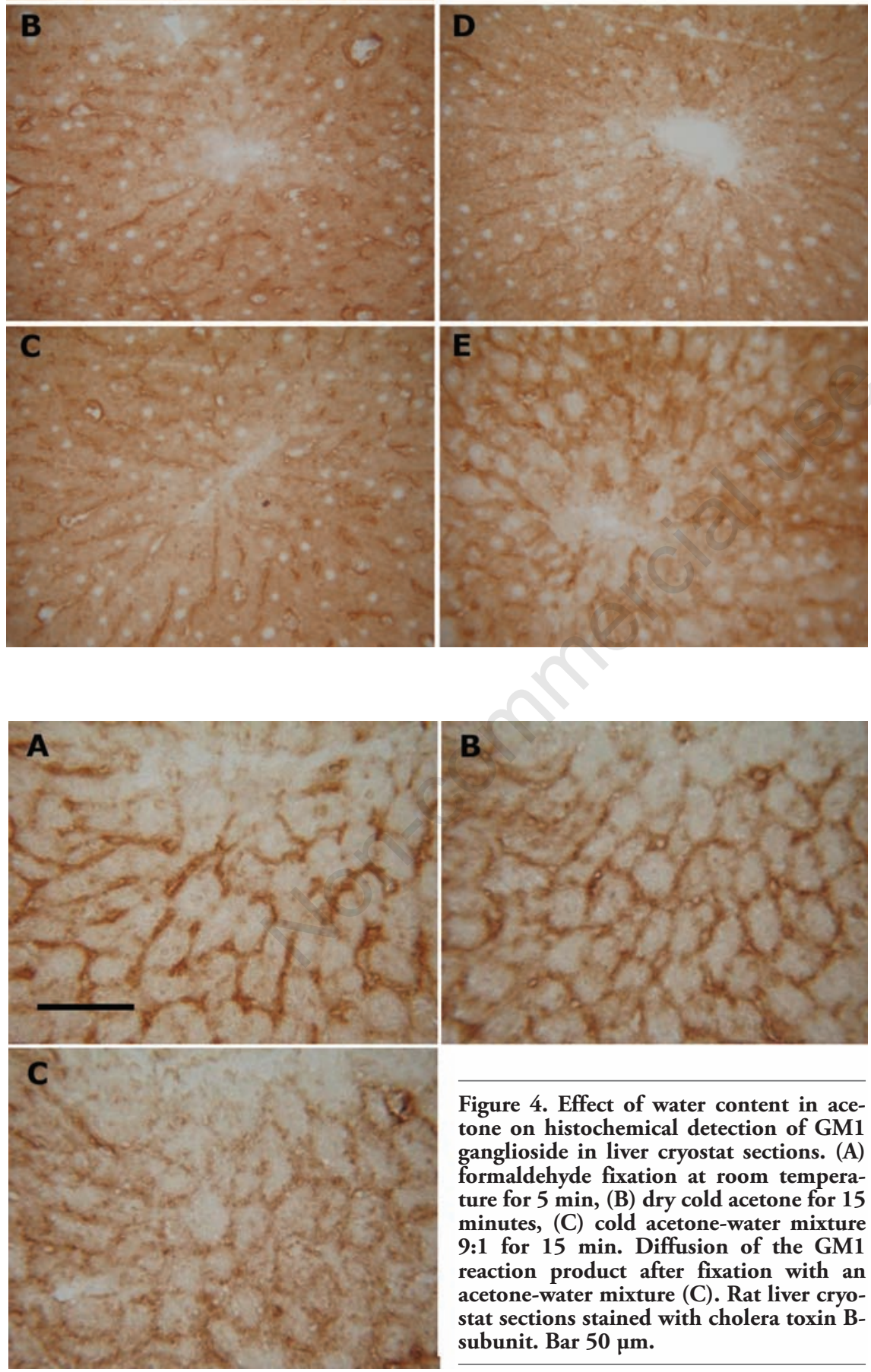

Figure 4. Effect of water content in acetone on histochemical detection of GM1 ganglioside in liver cryostat sections. (A) formaldehyde fixation at room temperature for $\mathbf{5} \mathrm{min},(\mathrm{B})$ dry cold acetone for $\mathbf{1 5}$ minutes, $(\mathrm{C})$ cold acetone-water mixture 9:1 for $15 \mathrm{~min}$. Diffusion of the GM1 reaction product after fixation with an acetone-water mixture (C). Rat liver cryostat sections stained with cholera toxin Bsubunit. Bar $50 \mu \mathrm{m}$.
GM1 staining were also examined. Liver sections fixed with dry acetone at $-20^{\circ} \mathrm{C}$ or $25^{\circ} \mathrm{C}$, both for 2 and $15 \mathrm{~min}$, were compared with those fixed with formaldehyde at room temperature for $5 \mathrm{~min}$. No apparent difference in the staining intensity and/or location of the reaction product was observed in corresponding areas of parallel sections (Figure 3). On the contrary, fixation with an acetone-water mixture caused apparent diffusion of the GM1 reaction product (Figure 4).

\section{Densitometric analysis of GM1 staining in liver sections}

Densitometry was used for evaluation, in order to obtain objective data regarding the optical density of GM1 staining in tissue sections. The differences in the optical density of GM1 staining in liver tissue from 23 normal animals after formaldehyde and anhydrous acetone fixation were not statistically significant (data not shown). Finally, neither formaldehyde nor dry acetone fixation caused diffusion of the reaction product in the liver sections.

\section{Comparison of histochemical detection of GM1 ganglioside in liver and brain cryostat sections after different fixations}

Unlike the liver samples, where formaldehyde and acetone fixations of cryostat sections gave minimal differences, different pretreatment of brain sections led to remarkably different GM1 staining patterns. Cryostat brain sections fixed with formaldehyde (F) displayed a diffuse GM1 staining pattern in the neuropil of cortical and subcortical grey matter, without focal accentuation of the staining (Figure $5 \mathrm{~A}, \mathrm{C}$ ). The slight and diffuse positivity seen in the white matter probably corresponds to myelin. Baker's solution (B) used instead of formaldehyde (F) had no apparent effect on the diffuse character of GM1 staining. After fixation with anhydrous acetone followed by formaldehyde $(\mathrm{A}+\mathrm{F})$, overall GM1 staining was less intensive but the staining pattern became much more distinct, revealing the presence of definable subcellular structures (Figure 5B,D). The finely granular GM1 staining changed for coarsely granular with the anhydrous acetone plus Baker's solution (A+B) sequence (not shown).

Treatment of brain sections with MCD led to decreased GM1 staining intensity against formaldehyde fixation (Figure $5 \quad \mathrm{G}, \mathrm{H}$ ). However, the sharp, focally accented staining pattern was less expressed than after acetone $(\mathrm{A}+\mathrm{F})$ as demonstrated (Figure $5 \mathrm{H}, \mathrm{I})$.

In the liver, the distinct, finely granular 
GM1 staining of hepatocyte membranes at both sinusoidal and canalicular poles did not display any substantial difference in sections fixed either in formaldehyde or in anhydrous acetone (Figure $5 \mathrm{E}, \mathrm{F}$ ).
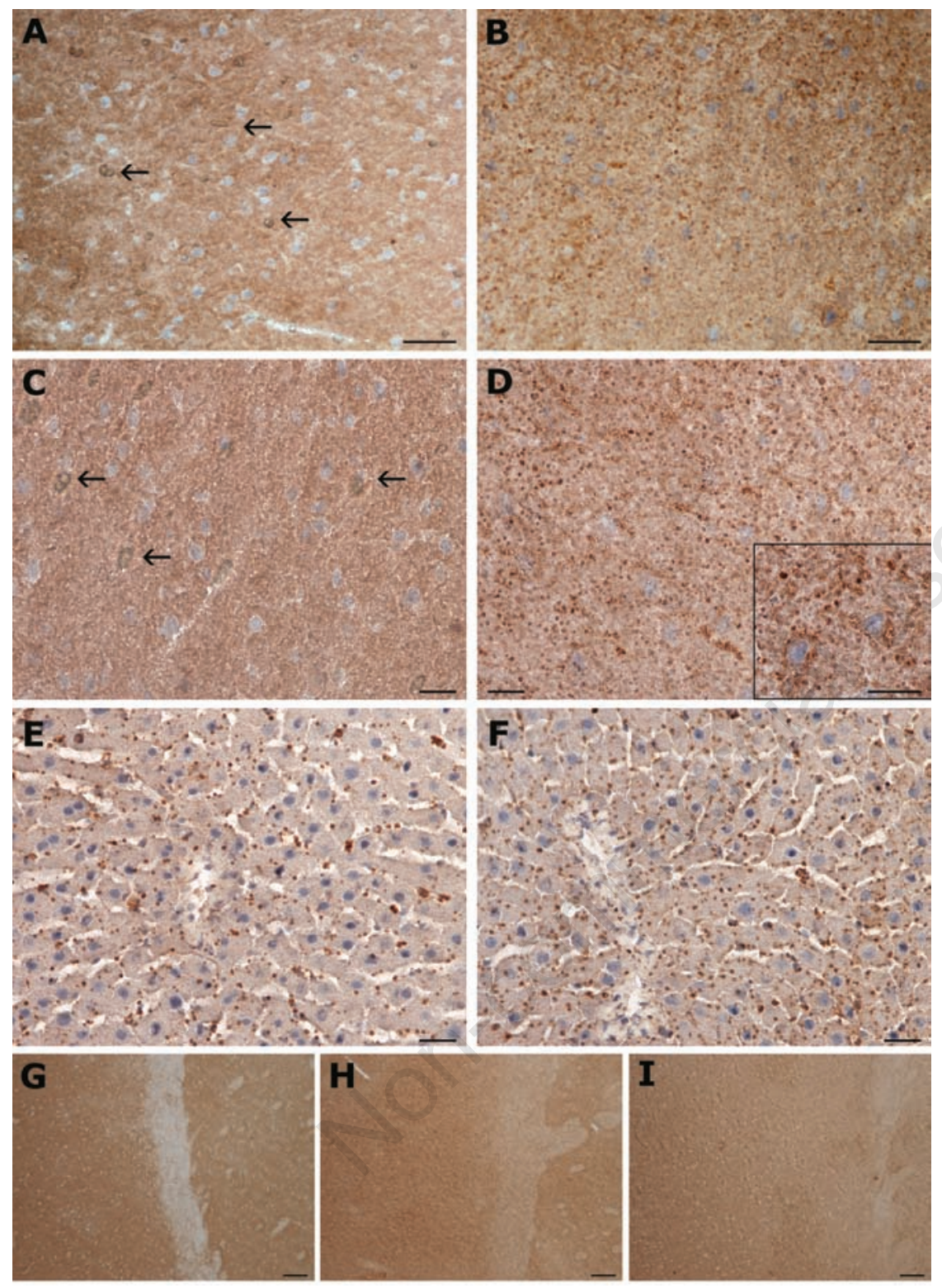

Figure 5. Compared effect of different fixatives on histochemical detection of GM1 ganglioside in rat liver and brain. Cryostat sections stained with cholera toxin B-subunit. (A, B) Brain cortical grey matter; low power field microphotographs. (A) Diffuse positivity in the neuropil after formaldehyde fixation and (B) focally accented staining after anhydrous acetone + formaldehyde $(A+F)$ combination. Bars $50 \mu \mathrm{m}$. $(C, D)$ Brain cortical grey matter; high power field microphotographs. (C) Diffuse staining in the neuropil after formaldehyde fixation. Note also a migration of unstained myelin lipids forming myelin figures $(A, C)$; some of them are marked by arrows. (D) Granular staining after acetone fixation $(A+F)$ suggesting localization of a reactive product to the cell membranes (for a detail see insert in D). Bars $25 \mathrm{\mu m}$. (E, F) High power field pictures of the liver sections fixed with (E) formaldehyde or $(\mathrm{F})$ acetone + formaldehyde $(\mathrm{A}+\mathrm{F})$. Finely granular staining on the cell membranes of hepatocytes is uninfluenced by the type of fixation. Bars $25 \mu \mathrm{m}$. Cell nuclei were counterstained with hematoxylin. $(\mathrm{G}, \mathrm{H}, \mathrm{I})$ survey of brain cortex; pre-treatment of cryostat sections with (G) formaldehyde, (H) MCD + formaldehyde and (I) anhydrous acetone + formaldehyde. Slight gradual $(G \rightarrow I)$ decrease in overall staining intensity and increase in sharpness and condensation of the staining pattern. Bars $100 \mu \mathrm{m}$. acetone pre-treatment, which should ensure effective retention of gangliosides in situ, in other words acetone pre-treatment should not lead to a significant loss of the lipids; and ii) evaluation of all factors responsible for optimal in situ demonstration.

As far as the reliability of acetone pre-treatment is concerned, the quantitative analysis of GM1 ganglioside in extracts of liver sections showed a negligible loss. Likewise, densitometric evaluation of the reaction product of histochemical GM1 detection in liver sections carried out in formaldehyde fixed sections and sections pretreated with acetone did not show statistically significant differences. Loss of gangliosides by acetone extraction of dried liver homogenate at room temperature was negligible $(3.0 \pm 0.3 \%)$. An increased loss of gangliosides in brain up to $9.5 \pm 1.1 \%$ corresponds to the less intensive GM1 staining in brain cryostat sections.

It might be possible to see an analogy with the conclusions of Ilangumaran and Hoessli, ${ }^{20}$ who proposed that in rafts, the GSL-rich core of the microdomains persists depletion of cholesterol with methyl- $\beta$-cyclodextrin (MCD), while the cholesterol-rich annulus surrounding the core, containing as well some GM1, seems to be partially released by MCD. It might be that acetone similarly extracts some GM1 from cholesterol-rich areas. Further extensive studies will be necessary to support this hypothesis.

Our study also confirmed the risk of pretreatment with acetone; extraction must be carried out with anhydrous acetone; this observation was previously described. ${ }^{14}$ It should be stressed that anhydrous acetone and dry cryostat sections are both essential for in situ preservation of gangliosides. Breaking of any of these conditions leads to ganglioside dislocation in situ or directly to their extraction extra situ. This might explain the loss of gangliosides described by Schwarz and Futerman, ${ }^{15}$ who found an approximate $56 \%$ reduction of gangliosides after acetone fixation and a $46 \%$ reduction after formaldehyde-acetone fixation of cultivated hippocampal neurons.

Acetone extraction is recommended for better in situ detection of gangliosides in many studies. ${ }^{8,12}$

The enhanced staining intensity after acetone pre-treatment is explained by the improvement of permeabilization. ${ }^{21}$ Glycosphingolipid globotriaosylceramide was more accessible to verotoxin in kidney sections after acetone pre-treatment; an effect ascribed to the removal of exceeding cholesterol. Similar effects were seen after methyl- $\beta$-cyclodextrin. ${ }^{13,22}$ Schwarz and Futerman ${ }^{15}$ also demonstrated brighter labeling of gangliosides in cultivated hippocampal neurons after combined fixation with formaldehyde and acetone, despite significant gangliosides removal dur- 
ing pre-treatment (see also above).

The finely granular GM1 positivity seen in acetone $(\mathrm{A}+\mathrm{F})$ fixed sections could represent the combined effect of unmasking, due to cholesterol extraction and precipitation resulting from the low solubility of GSLs in acetone (difference from MCD). The increased granularity and contrast seen when the combination of anhydrous acetone plus Baker's solution $(A+B)$ was used for fixation, could be explained by the well known aggregation of gangliosides in presence of $\mathrm{Ca}^{2+}$ ions. ${ }^{23,24}$

Our results in brain samples showed better outcomes in situ detection of GM1 after acetone pre-extraction of dry cryostat sections (Figure 5A-D) which also inhibited the considerable diffusion of myelin lipids into the mounting medium in acetone unextracted sections.

This suggests that the benefits of acetone, as a process of physical fixation, are related to precipitation of polar lipids generally (inhibition of their local diffusion), and to removal of the possible masking effect of cholesterol.

However, we should be aware that cryostat section technique is inherently influenced by thawing and subsequent drying of the sections that might influence in situ localization of polar compounds, including gangliosides. The current study offers the optimal way to cope with this problem.

\section{Conclusions}

We present for the first time evidence that both pre-treatments (formaldehyde and acetone) provide good information on GM1 localization at the light microscopy level in liver sections. In the brain, the quality of GM1 detection was found to be higher in acetone pretreated sections; optimal conditions vary based on the lipid composition of the tissue. The benefits of acetone pre-treatment are linked to the use of anhydrous conditions.

\section{References}

1. Hakomori S. Glycosphingolipids in cellular interaction differentiation and oncogenesis. In: Kanfer JN and Hakomori S eds. Handbook of Lipid Research. Vol. 3.
Sphingolipid Biochemistry. Plenum Press, New York, London 1983, pp. 327-336.

2. Hakomori S. Glycosynapses: microdomains controlling carbohydrate-dependent cell adhesion and signaling. An Acad Bras Cienc 2004;76:553-72.

3. Sonnino S, Mauri L, Chigorno V, Prinetti A. Gangliosides as components of lipid membrane domains. Glycobiology 2007;17:1R13R.

4. Hakomori SI. Structure and function of glycosphingolipids and sphingolipids: Recollections and future trends. Biochim Biophys Acta 2008;1780:325-46.

5. Holmgren J. Receptors for cholera toxin and Escherichia coli heat-labile enterotoxin revisited. Prog Brain Res 1994;101:16377.

6. Fukuta S, Magnani JL, Twiddy EM, Holmes RK, Ginsburg V. Comparison of the carbohydrate-binding specificities of Cholera toxin and Escherichia coli heat-labile enterotoxins LTh-I, LHT-Ila, and LHT-IIb. Infect Immun 1988;56:1748-53.

7. MacKenzie CR, Hirama T, Lee KK, Altman A, Young NM. Quantitative analysis of acterial toxin affinity and specificity for glycolipid receptors by surface plasmon resonance. J Biol Chem 1997;272:5533-8.

8. Kotani M, Kawashima I, Ozawa H, Terashima T, Tai T. Differential distribution of major gangliosides in rat central nervous system detected by specific monoclonal antibodies. Glycobiology 1993;3:137-46.

9. Chen Y, Qin J, Chen ZW. Fluorescencetopographic NSOM directly visualizes peak-valley polarities of GM1/GM3 rafts in cell membrane fluctuations. J Lipid Res 2008;49:2268-75.

10. Schwarz A, Futerman AH. The localization of gangliosides in neurons of the central nervous system: the use of anti-ganglioside antibodies. Biochim Biophys Acta 1996;1286:247-67.

11. Schwarz A, Futerman AH. Immunolocalization of gangliosides by light microscopy using anti-ganglioside antibodies. Methods Enzymol 2000;312:179-87.

12. Kotani M, Kawashima I, Ozawa H, Ogura $\mathrm{K}$, Ishizuka I, Terashima T et al. Immunohistochemical localization of minor gangliosides in the rat central nervous system. Glycobiology 1994;4:855-65.

13. Chark D, Nutikka A, Trusevych N, Kuzmina J, Lingwood C. Differential carbohydrate epitope recognition of globo- triaosyl ceramide by verotoxins and a monoclonal antibody. Eur J Biochem 2004;271: 405-17.

14. Elleder M, Lojda Z. Studies in lipid histochemistry. VI. Problems of extraction with aceton in lipid histochemistry. Histochemie 1971;28:68-87.

15. Schwarz A, Futerman AH. Determination of the localization of gangliosides using anti-ganglioside antibodies: comparison of fixation methods. J Histochem Cytochem 1997;45:611-8.

16. Ledeen RW, Yu RK, Eng LF. Gangliosides of human myelin: sialosylgalactosylceramide (G7) as a major component. J Neurochem 1973;21:829-39.

17. Svennerholm L. Quantitative estimation of sialic acids. II. A colorimetric resorcinolhydrochloric acid method. Biochim Biophys Acta 1957;24:604-11.

18. Jirkovská M, Majer F, Šmídová J, Stríteský J, Shaik GM, Dráber P et al. Changes in GM1 ganglioside content and localization in cholestatic rat liver. Glycoconj J 2007; 24:231-41.

19. Ellerbe P, Meiselman S, Sniegoski LT, Welch MJ, White E. Determination of serum cholesterol by a modification of the isotope dilution mass spectrometric definitive method. Anal Chem 1989;61:1718-23.

20. Ilangumaran S, Hoessli D. Effects of cholesterol depletion by cyclodextrin on the sphingolipid microdomains of the plasma membrane. Biochem J 1998;335:433-40.

21. Kusunoki S, Chiba A, Hirabayashi Y, Irie F, Kotani M, Kawashima I, et al. Generation of a monoclonal antibody specific for a new class of minor ganglioside antigens, GQ1b alpha and GTla alpha: its binding to dorsal and lateral horn of human thoracic cord. Brain Res 1993;623:83-8.

22. Khan F, Proulx F, Lingwood CA. Detergentresistant globotriaosyl ceramide may devone verotoxin/glomeruli-restricted hemolytic uremic syndrome pathology. Kidney Int 2009;75:1209-16.

23. Sharom FJ, Grant CW. Glycosphingolipids in membrane architecture. J Supramol Struct 1977;6:249-58.

24. Sharom FJ, Grant CW. A model for ganglioside behaviour in cell membranes. Biochim Biophys Acta 1978;507:280-93. 